\title{
Critical care/Emergency Lab
}

Cod: 0436

COMPARISON OF CRP MEASUREMENT OF THE MICROSEMI® WITH THE MICROS CRP® AND VITROS ${ }^{\circledR} 5600$ ANALYSERS

H. Baum $^{1}$, H. Sander ${ }^{1}$, A. Leni ${ }^{1}$, K. Mages $^{1}$

${ }^{1}$ Regionale Kliniken Holding RKH GmbH

BACKGROUND: CRP is an important parameter in the diagnosis and follow-up of patients with infections. To improve patient care in an acute setting it will be more and more important to reduce blood drawn and to get the results very fast, without affecting quality of results. The Microsemi analyser needs $18 \mu$ to measure a blood cell count and CRP within 4 minutes. In this study we compared the CRP determination of the new released Microsemi in serum and EDTA-blood with the Micros CRP and Vitros 5600 analysers.

METHODS: For comparison we used 100 samples of serum and EDTA-blood out of the daily routine from patients in whom both specimens were drawn and CRP was ordered. Both samples from each patient were measured on the Microsemi and Micros CRP in duplicate. We compared the results of all three analysers with each other as well as the results of the serum and EDTA-blood samples of the Microsemi and Micros CRP analysers.

RESULTS: The comparison of the different specimens showed that there was a good concordance between the Micros analysers. Concerning the CRP measure, the comparison of the two Micros analysers, the Microsemi showed all about $35 \%$ higher CRP values. However, when the Micros analysers were compared to the Vitros 5600, this latest one showed similar results versus the Microsemi but lower values versus the Micros CRP of $20 \%$ in serum and $26 \%$ in EDTA-blood.

CONCLUSIONS: The new Microsemi is an accurate and fast analyser to measure CRP in whole blood or serum. It shows a very good comparability to a standard laboratory method with both, serum or EDTA-blood. Also it is superior to the older Micros CRP analyser. Therefore, it can be used as a real alternative for single high priority specimen to enhance the turn-around-time in this specimen without loss of accuracy. 


\title{
Critical care/Emergency Lab
}

Cod: 0437

\section{COMPLETE BLOOD COUNT (CBC) IN THE EMERGENCY DEPARTMENT; PRE-ANALYTICAL ISSUES FOR HEMOGLOBIN AND} LEUKOCYTES

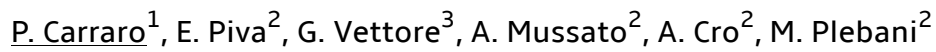 \\ ${ }^{1}$ Department of Laboratory Medicine, St. Antony Hospital, Padova, Italy \\ ${ }^{2}$ Department of Laboratory Medicine, University Hospital, Padova, Italy \\ ${ }^{3}$ Emergency Department, University-Hospital, Padova, Italy
}

BACKGROUND: The pre-analytical conditions that affect intra-individual fluctuations of variables related to $C B C$ in patients admitted to the Emergency Department (ED) could be functions of waiting time in an orthostatic posture, lack of fasting, or intense psychological stress. The impacts of these factorss have not previously been studied in the particular context of an ED.

METHODS: We analyzed the differences in Hemoglobin (HGB) and Leukocyte (WBC) count of all CBCs ordered by a large ED over a period of one year, comparing the data with a second CBC carried out within 24 hours for reasons of care and diagnosis ( 11,487 cases). In order to identify the factors that influence the changes we collected clinical data for a consecutive series of 1025 cases with similar conditions, recording: body temperature, pretibial pitting edema, blood or transfused components, cortisone for systemic use, diuretics, infusion of fluids. A multivariate analysis was thus obtained.

RESULTS: The differences between the two subsequent CBC determinations for all patients considered in a year (excluding values $<50$ or $>160 \mathrm{~g} / \mathrm{L}$ ), demonstrated a mean fall of $\mathrm{HGB}$, in the second test of $2.8-8.7 \mathrm{~g} / \mathrm{L}$ (3.0-6.3\%) (Wilcoxon signed-rank test, $\mathrm{p}<0.001)$. The WBC count was also found to decrease in the second examination, but only for results $>8.0 \times 10^{9} / \mathrm{L}$ : the median difference ranging from 0.75 to $3.59 \times 10^{9} / \mathrm{L}(8-20 \%)(\mathrm{p}<0.001)$. The multivariate analysis performed in the abovementioned 1025 cases, showed a significant effect on the HGB variation when the volume of infused fluids were taken into consideration, especially if the latter was $>500 \mathrm{ml}$ in the first 24 hours (Kruskal-Wallis test, $p=0.001$ ). The changes in WBC were not significantly different in the various groups.

CONCLUSIONS: Our data confirm important changes in HGB concentration in an ED context, which are likely to depend upon the hemoconcentration at the time of the check in. This is particularly relevant if the patient is monitored for suspected internal bleeding. The WBC count is increased when the patient at the first test presents values $>8.0$ that could presumably be attributable to stress and anxiety levels. These test results need to be assessed with caution in cases of suspected infection or sepsis. 


\title{
Critical care/Emergency Lab
}

Cod: 0439

THE ROLE OF THE LAB IN THE OPERATIONAL PLANNING OF THE FIRST AID SERVICE

\author{
$\underline{\text { F. Facco }}^{1}$, S. Mangraviti ${ }^{1}$, R. Cozzani ${ }^{1}$, R. Pessina ${ }^{1}$, M. Garaventa ${ }^{1}$, P.G. Montaldo ${ }^{1}$ \\ ${ }^{1}$ Department of Translational Research and Laboratory Medicine, G. Gaslini Children Hospital, Genova, Italy
}

BACKGROUND: Urgency classification at the first aid service is mainly made on a clinical basis (triage) without considering any other diagnostic tool such as laboratory tests. This might underestimate the need for first aid human and facility resources more suitable to face the increased needs of the population. In this retrospective report we analyze data about the triage code assigned by the first aid emergency service and the request of laboratory analyses.

METHODS: In our Hospital, over a 25 years period, overall first aid admission increased from 7000 to 22600 patients (median increase $6 \%$ / year). At the triage the red and yellow codes (the critical or the most serious cases) were $10 \%$, whereas $60 \%$ of children were classified as a low priority (green code) and $30 \%$ not urgent at all (white code). Nevertheless, in addition to all patient with red and yellow code (2200 pts), laboratory test were required for $18 \%$ of children (3650 pts) classified as a green and white code. Each analytical panel included an average of 14 test for each patient.

RESULTS: This resulted in a no-stop involvement of the diagnostic service for more than 80000 tests.

Considering the operational involvement other than clinical criteria only, the percent of incoming patients that activated the "urgency system" switched from $10 \%$ to $25 \%$. We assume that critical situations were immediately identified and properly coded at the first aid service. Perhaps a considerable percent of patients fall in a grey zone or might have been miscoded on a clinical basis only.

CONCLUSIONS: In a modern health system the first aid pediatrician needs the most precise criteria to make a proper admission diagnosis or a safe discharge of the patient, even for the sake of the community (eg. contumacy for diffusive diseases). The laboratory of analysis can thus provide useful operational and epidemiological data to redefine the real entity of urgency in an operative scenario. This can contribute to an optimal planning of "ready to answer" services according to the growing pressure on the first aid by the population. 


\section{Critical care/Emergency Lab}

Cod: 0440

STABILITY STUDY OF BILIRUBIN IN CSF STORED AT FOUR DEGREES CELSIUS AND IN THE ABSENCE OF LIGHT

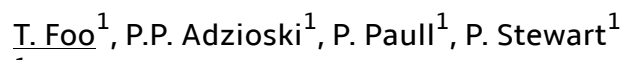

${ }^{1}$ Dept. of Clinical Biochemistry, Royal Prince Alfred Hospital, Sydney

BACKGROUND: Operational considerations may require overnight storage of CSF samples before analysis for Xanthochromia. There have been a number of studies that have assessed the stability of Bilirubin in CSF but they have not specifically addressed stability of Bilirubin close to the cut-off concentration as specified in the UK National Guidelines for the presence of Xanthochromia (Ann Clin Biochem 2008;45:238-44). The present study was designed to assess stability of Bilirubin in CSF stored at 4 degrees Celsius in the dark and at concentrations close to the cut-off.

METHODS: Sixteen pools of CSF were prepared from samples taken at lumbar puncture. Each pool was spiked with a human serum that had a high concentration of Bilirubin. The serum added did not exceed $0.1 \%$ of the volume of the CSF pool. The spiked pools had Bilirubin concentrations that produced absorbances between 0.0082 and 0.0106 as defined in the UK National Guidelines. These guidelines define a Net Bilirubin Absorbance (NBA) of $\leq 0.007 \mathrm{AU}$ at $476 \mathrm{~nm}$ as negative for Xanthochromia that arises from subarachnoid haemorrhage. No patient was used more than once for the CSF pool or as a source of bilirubin. Samples were stored at 4 degrees Celsius in the dark and Bilirubin assayed immediately before storage and after 16 hrs of storage. NBA was measured on an Agilent Cary 60 spectrophotometer. A Student's Paired t-Test was used to assess the statistical significance of differences in the mean initial NBA and the mean NBA determined after 16 hrs of storage. Absorbance values measured using the Cary spectrophotometer are accurate and precise. The measurement of NBA for a commercial material had a mean value of 0.0101 ( $n=16$, assigned value $0.0100 \mathrm{AU})$. Intra-assay imprecision was a CV of $1.9 \%(n=12)$. Inter-assay imprecision was a CV of $4.2 \%(n-=16)$.

RESULTS: The mean initial NBA was $0.0093(S D=0.0010, n=16)$. The mean NBA after 16 hours of storage was 0.0094 $(S D=0.0010)$. There was no significant difference between the mean NBA before storage and the mean NBA determined after storage for $16 \mathrm{hrs}$.

CONCLUSIONS: This study shows that following proper storage of CSF samples there will be no affect on the determination of Xanthochromia even when the Bilirubin concentration are close to the cut-off NBA. 


\section{Critical care/Emergency Lab}

Cod: 0441

\section{CRITICAL VALUE REPORT SYSTEM IMPLEMENT IN A LARGE UNIVERSITY-BASED HOSPITAL}

\section{S.J. Ok ${ }^{1}$, S.M. Lee ${ }^{2}$}

${ }^{1}$ The Department of Laboratory Medicine, Pusan National University Hospital, Busan, Republic of Korea

${ }^{2}$ The Department of Laboratory Medicine, Pusan National University Yangsan Hospital, Yangsan, Gyeongnam, Republlic of Korea

BACKGROUND: Worldwide, there are too many test results released to clinicians with rapid turnaround times to communicate and follow-up on the clinically significant test results. To improve patient safety and communication systems, our hospital developed a critical laboratory result management system. The aim of this study was to evaluate the usefulness of this new system and monitor the length of time between verification and notification of critical value.

METHODS: The task force developed and implemented the critical result communication infrastructure, named the Critical Value Report (CVR) system, with automated alternatives in our electronic medical record system (EMR). The CVR system includes multiple time parameters, including the time of sample acceptance, verified result time, time elapsed before reaching a nurse, transmission time to doctor, and the time the result was checked by physician on the EMR. Therefore, anyone can assess the length of time for the delivery and receipt of critical value category test results. To evaluate the CVR system that implemented in large university based hospital staffed by the over 1,000 caregivers, we reviewed the critical values and CVRs and followed the reporting times, using the records from December 4, 2013, to February 4, 2014.

RESULTS: During the period of the study, excluding microbiology and blood bank, 3,254 test results $(0.3 \%$ of total test results) were deemed as critical value. The laboratories reported 1,682 critical values after automated filtering algorithm. Therefore, tests with critical values represented approximately $0.07 \%$ of the total test results. Most CVRs $(87.3 \%)$ included one of eight typically significant tests. The majority of critical values resulted from testing performed in the hematology (38.5\%) laboratory, followed by in the coagulation (32.4\%) and clinical chemistry (21.4\%) laboratory. After implementing the CVR system on the EMR, the number of phone calls per week was reduced by $56 \%$. The time when the critical value information was conveyed to clinician was different depending on the patient's locations.

CONCLUSIONS: The CVR system on the EMR effectively transmitted critical value test results, so it would contribute to patient safety. 


\title{
Critical care/Emergency Lab
}

Cod: 0442

AN AUTOMATED MESSAGING SERVICE FOR THE REPORTING OF CRITICAL ROUTINE LABORATORY RESULTS. A RETROSPECTIVE ANALYSIS

\author{
M.F. Aumentado $^{3}$, Y.H. Chew ${ }^{2}$, P.B. Liew ${ }^{2}$, L.L. Phoa ${ }^{3}$, M.S. Wong ${ }^{3}$, S. Tsai ${ }^{1}$, M. Sellathambi ${ }^{1}$ \\ ${ }^{1}$ Department of Laboratory Medicine, Khoo Teck Puat Hospital \\ ${ }^{2}$ Integrated Health Information Systems, Khoo Teck Puat Hospital \\ ${ }^{3}$ Khoo Teck Puat Hospital
}

BACKGROUND: A critical laboratory result has limited usefulness until it is put into the hands of a person who can interpret it and implement the appropriate remedial actions in the fastest possible time. In a manual callback process of critical results, the lag time between the availability of the data and the ultimate receipt by the physician in charge is dependent upon several factors and in almost all cases, the relaying of data between the laboratory personnel and the nurse. With the fully automated Health Messaging System (HMS), the physician is notified directly by short messaging service, which incorporates a three reply option and automated escalation.

METHODS: In the manual callback process, the callback audit details were documented in the Laboratory Information System (LIS). The LIS database was mined for tests that were defined as having critical values, test resulting time and telephone comment closure. Patient details were not required in this study. Similarly, the automated HMS database was mined to provide comparable details.

RESULTS: From March 2010 to August 2013, 4951 and 13,332 critical results were handled by the laboratory and HMS respectively. The average and median closure times were 11 and 4 minutes for manual callback and 12 and 7 minutes for HMS respectively. The manual callback process closed $54 \%$ and $92 \%$ of cases at 5 minutes and 30 minutes respectively while HMS closed $52 \%$ and $93 \%$ at the same time points. At 90 minutes, $99.6 \%$ and $99.7 \%$ of cases were communicated by the HMS and manual systems respectively. For inpatient data alone, HMS outperformed the manual system by between $1 \%$ and $7 \%$ at most time points. In the Emergency department, the HMS appears to perform poorly by as much as $23 \%$ at 10 minutes, but recovers at the 60 minute mark.

CONCLUSIONS: Both the manual and automated critical result reporting processes appear comparable in performance. However, the automated system is deemed to be superior as only $5 \%$ of cases were actually conveyed to doctors by the manual system. The time taken to relay the information is an unknown quantum but will contribute additional time to the communication process. 


\title{
Critical care/Emergency Lab
}

Cod: 0443

\section{EVALUATION OF SERIAL MONITORING OF PCT LEVELS AS PROGNOSTIC BIO MARKER OF SEPSIS}

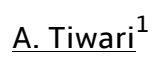 \\ ${ }^{1}$ Care Hospitals, The Institute of Medical Sciences
}

BACKGROUND: Procalcitonin (PCT) is a116 amino acid precursor of calcitionin (CT).During severe sepsis in particular, related to bacterial infection (B I) it is secreted in large quantities by body tissues, contribute to sepsis related death. PCT levels are detectable in 2-4 hrs after BI and peaks by 6-24 hrs. This retrospective observational study evaluates serial monitoring of PCT as prognostic bio marker of sepsis, to assist the clinicians for diagnosis and treatment .

METHODS: PCT levels were monitored for patients $(\mathrm{N}=2055)$ admitted with $\mathrm{H} / \mathrm{O}$ fever and infection. Patients were grouped (gp)as $(A=<0.5),(B=\geq 0.5-<2.0),(C=\geq 2.0-<10.0),(D=\geq 10-<50.0),(E=\geq 50-<100)(F=\geq 100.0)$ as per PCT levels. PCT were analysed on DOA (T1) and monitored after treatment on T2, T3, and T4 up to T10 times. Each time gp A was excluded from repeat testing.

RESULTS: On T1 patients ( $\mathrm{N}=2055$ mean 6.86 )grouped as( $\mathrm{A}-32 \% \mathrm{~B}-24 \% \mathrm{C}-23 \% \mathrm{D}-13 \% \mathrm{E}-3.0 \% \mathrm{~F}-4.5 \%)$. On T2 ( $\mathrm{N}=1417$ mean5.8) trends were (A-38\%B-23\%C-20\%D-11\%E- 2\% F-4.4\%). Patients showed declining trends in PCT levels, and also in the number of patients in each gp except gp A. On T3 ( $N=373$ mean 8.8) trends observed (A-22\%B-26\%C-27\%D-17\% E-3.7\% F-4.2\%). Treatment reviewed for gps C D E F. On T4 (N=193mean 10.8) trends seen (A- 40\% B-18\%C-9\% D-11\% E-5.6\% F-4.6\%). Treatment failure(TF) / secondary infection (SI) in gps C D E F. On T5 (N=63 mean11.3) trends seen (A-6.3\% B-27\%C-41\%D-19\% E- 4.7\%F- 4.6\%). TR for C D E F gps. On T6 (N= 28 mean 13.4) trends seen $(\mathrm{A}-3.6 \% \mathrm{~B}-11 \% \mathrm{C}-43 \% \mathrm{D}-28 \% \mathrm{E}-3.6 \% \mathrm{~F}-10 \%)$. SI in gps C D E \& high mortality rate seen in gp $\mathrm{F}$. OnT7 (N=15 mean 12.2) trends seen (A-6.6\% B-20\%C-40\%D-26\%E-6.6\%).TF for CDEFgps. No declining trends seen in gPs CDEF for $\mathrm{T} 8(\mathrm{~N}=9$ mean13.2), T9 $(\mathrm{N}=4$ mean13.5) \&T10 ( $\mathrm{N}=3$ mean14.6).

CONCLUSIONS: Higher PCT levels associated with more severe diseases making it suitable for serial monitoring. Declining levels are associated with resolution of illness as the infection resolve, PCT were eliminated in 24-36 hrs. PCT levels not declining or increasing showed strong negative prognostic indicator of lack of control of the infection. PCT levels were declining in a predictable pattern in the absence of SI. Higher mortality rate were observed with consistently higher PCT levels (>100). 